a. Servicio de Errores Congénitos del Metabolismo, Hospital "Prof. Dr. Juan P. Garrahan".

b. Servicio de Neurología Infantil, Clínica de Mucopolisacaridosis, Hospital Universitario Austral, Pilar, Buenos Aires.

Oftar, Buenos Aires.

Universidad Católica Argentina. Sección Genética Médica, Hospital de Niños Dr. Ricardo Gutiérrez, Buenos Aires.

e. Hospital Privado de Córdoba

Endoscopía Respiratoria Pediátrica, Hospital “Prof. Dr. Juan P. Garrahan".

g. División Cardiología, Hospital de Niños Dr. Ricardo Gutiérrez.

Sección de Audiología, Servicio de Otorrinolaringología,

Hospital de Niños Dr. Ricardo Gutiérrez.

Gección Reumatología

Sección Reumatología Pediátrica, Servicio Clínica Pediátrica, Departamento de Pediatría, Hospital Italiano Buenos Aires.

j. Servicio de Hematología Hospital de Niños Dr. Ricardo Gutiérrez y Fundación Favaloro.

k. Servicio de Reumatología Infantil, Hospital de Niños Dr. Ricardo Gutiérrez.

Dr. Ricardo Gutiérrez.
Servicio de Errores Congénito del Metabolismo, Hospital "Prof. Dr. Juan P. Garrahan".

. Coordinador del Hospital de Día Polivalente, Hospital de Niños Dr. Ricardo Gutiérrez.

m. Servicio de Crecimiento y Desarrollo, Hospital “Prof. Dr. Juan P. Garrahan".

n Servicio de Enfermedades Metabólicas, Clínica Universitaria Reina Fabiola

Centro Respiratorio, Hospital d Niños Dr. Ricardo Gutiérrez.

Secciones Alergia Pediátrica y Adultos, Hospital Italiano de Buenos Aires. Coordinador del Grupo de Trabajo Alérgenos Alimentarios, International Life Sciences Institute (ILSI) Argentina.

p. Crecimiento y Desarrollo, referente en Errores Congénitos del Metabolismo de Mendoza, Hospital Pediátrico Hospital Pediátrico
Dr. H. J. Notti de Mendoza. Patología Espinal, Hospital Patología Espinal, Hospital
"Prof. Dr. Juan P. Garrahan" "Prof. Dr. Juan P. Garrahan"

Laboratorio Dr. Chamoles. Hospital Sor María Ludovica, La Plata.

t. Servicio de Trasplante de Medula Ósea, Hospital "Prof. Dr.Juan P. Garraha".

Neurocirugía Universidad

der

de Buenos Aires (UBA)

Servicio de Neurocirugía,

Gospital "Prof. Dr. Juan P.

Garrahan", ex presidenta de la

International Society of Pediatric
Neurosurgery (ISPN), medallista de Federación Latinoamericana de Sociedades de Neurocirugía (FLANC), 2018.

Correspondencia:

Dra. Luisa Bay: bay.luisa@gmail.com

Financiamiento: Ninguno.

Conflicto de intereses:

Los doctores Luisa Bay, Norberto Guelbert, Alejandro Fainboim

Norma Spécola, Guillermo

Drelichman y Marcela Pereyra han recibido honorarios del laboratorio Sanofi por tareas docentes sobre enfermedades lisosomales.

Recibido: 21-7-2020 Aceptado: 20-10-2020

\title{
Nuevas recomendaciones para el cuidado de los pacientes con mucopolisacaridosis tipo I
}

\author{
New recommendations for the care of patients with \\ mucopolysaccharidosis type I
}

Dra. Luisa Bay ${ }^{a}$, Dr. Hernán Amartino ${ }^{b}$, Dra. Alejandra Antaclec, Dra. Claudia Arberas ${ }^{d}$, Dra. Adriana Berretta ${ }^{e}$, Dr. Hugo Bottof, Dra. Mariana Cazalass, Lic. Adriana Copizh,

Dra. Carmen De Cunto ${ }^{i}$ Dr. Guillermo Drelichmani, Dra. Graciela Espada ${ }^{k}$, Dr. Hernán Eiroal, Dr. Alejandro Fainboim ${ }^{l l}$, Dra. Virginia Fano ${ }^{m}$, Dr. Norberto Guelbern, Dr. Alberto Maffey ${ }^{\tilde{n}}$, Dr. Claudio Parisi ${ }^{\circ}$, Dra. Marcela Pereyra ${ }^{p}$, Dr. Rodrigo Remondino, Farm. Andrea Schenone ${ }^{r}$, Dra. Norma Spécolas, Dra. Raquel Staciuk ${ }^{t} y$

Dra. Graciela Zuccarou

\section{RESUMEN}

Dados los avances sobre mucopolisacaridosis I con posterioridad al consenso publicado en la Argentina por un grupo de expertos en 2008 , se revisan recomendaciones respecto a estudios genéticos, seguimiento cardiológico, cuidado de la vía aérea, alertas sobre aspectos auditivos, de la patología espinal y neurológica. Se hace revisión de la terapéutica actual y se enfatiza en la necesidad de un diagnóstico y tratamiento precoces, asícomo de un seguimiento interdisciplinario.

Palabras clave: mucopolisacaridosis I, clasificación, terapéutica, diagnóstico, seguimiento.

http:/ / dx.doi.org/10.5546/ aap.2021.e121

Texto completo en inglés:

http: / / dx.doi.org/10.5546/ aap.2021.eng.e121

Cómo citar: Bay L, Amartino H, Antacle A, Arberas C, et al. Nuevas recomendaciones para el cuidado de los pacientes con mucopolisacaridosis tipo I. Arch Argent Pediatr 2021;119(2):e121-e128.

\section{INTRODUCCIÓN}

En 2008, se publicó un consenso de diagnóstico y tratamiento de mucopolisacaridosis tipo I (MPS I) en la Argentina. ${ }^{1} \mathrm{El}$ tiempo transcurrido con respecto al tratamiento y al seguimiento en nuestro medio y en el mundo lleva a revisar las recomendaciones sobre algunos aspectos de la enfermedad.

\section{Formas clínicas}

Los pacientes con MPS I se clasificaban según su gravedad en tres formas clínicas: Hurler, Hurler-Scheie y Scheie. Actualmente, se define como forma grave (FG) lo que era S. Hurler y forma atenuada (FA) abarca las anteriores H. Scheie y Scheie. ${ }^{1}$

\section{Genética}

La MPS I es una enfermedad a utosómica recesiva cuya heterogeneidad genética explica, en parte, la heterogeneidad clínica. El gen responsable es IDUA con 14 exones, locus $4 p 16.3$. Se conocen unas 100 mutaciones, y 20 de ellas cubren entre el $85 \%$ y el $90 \%$ de los casos.

En el 90-95\% de los casos, se identifican las mutaciones por secuenciación completa, pues técnicas más simples pueden no hacerlo. Hay mutaciones missense (en FA), nonsense (en FG) o por deleción.

En la FG, las mutaciones más frecuentes son W402X (el $45 \%$ ) y Q70X (el $16 \%$ ). Ambas en homocigosis. En la FA, suele encontrarse heterocigotas compuestos con una de ellas.

La forma clínica no se determina por el dosaje enzimático ni por marcadores bioquímicos. La correlación genotipo/ fenotipo puede ayudar, especialmente, en el recién nacido.

Sobre 538 MPS I del registro internacional, el $67,6 \%$ de los 380 FG 
tenían mutaciones que permitían predecir el fenotipo. ${ }^{2}$ Todo paciente diagnosticado debe recibir asesoramiento genético.

\section{Evaluación cardiológica}

El 88-93\% de los pacientes con FG y FA tienen manifestaciones cardíacas ${ }^{3,4}$ (Tabla 1). Los tratamientos disponibles aumentan la sobrevida, pero la enfermedad coronaria y las valvulopatías se presentan a pesar del tratamiento ${ }^{5,6}$ y causan elevada morbimortalidad. El $50 \%$ de los pacientes con FG mueren por causa cardíaca.-9

Se informaron anomalías valvulares en el $35 \%$ de los pacientes latinoamericanos con FG y en el $54 \%$ en las FA, versus el $73 \%$ y el $87 \%$ en los del resto del mundo. Será por un fenotipo regional o por la forma de evaluación diagnóstica. ${ }^{10}$

Se recomienda lo siguiente para el diagnóstico y el seguimiento cardiológico:
- Examen cardiovascular, electrocardiograma (para alteraciones del ritmo) y ecocardiogramas iniciales.

- En el seguimiento: ${ }^{11-13}$

Ecocardiograma cada 1-2 años si no hay alteraciones o estas son leves.

Cada 6 meses o más frecuente cuando hay afecciones de moderadas a graves.

- Holter, solo indicado ante palpitaciones o arritmias en el electrocardiograma.

- Test de ejercicio cardiopulmonar; difícil de realizar por las alteraciones esqueléticas; sin estudios que avalen su práctica.

- Por sospecha de coronariopatía, se hace tomografía computada (TC) multislice, perfusión miocárdica y angiotomografía o angiorresonancia con sospecha de afección de grandes arterias.

TABLA 1. Las manifestaciones cardíacas más importantes

\begin{tabular}{|c|c|c|c|c|}
\hline $\begin{array}{l}\text { Afección } \\
\text { cardíaca }\end{array}$ & Frecuencia & Manifestación & $\begin{array}{c}\text { Diagnóstico } \\
\text { y seguimiento }\end{array}$ & $\begin{array}{l}\text { Modificaciones } \\
\text { con tratamiento }\end{array}$ \\
\hline $\begin{array}{l}\text { Valvulopatía } \\
\text { mitral/aórtica }\end{array}$ & $\begin{array}{l}\text { Más frecuente la } \\
\text { afectación mitral. }\end{array}$ & $\begin{array}{l}\text { Insuficiencia } \\
\text { y estenosis. }\end{array}$ & $\begin{array}{l}\text { Ecocardiograma (el } 57 \% \text { tienen } \\
\text { auscultación normal). } \\
\text { El } 14 \% \text { de las formas atenuadas } \\
\text { requieren reemplazo valvular } \\
\text { en evolución. }\end{array}$ & $\begin{array}{l}\text { El TCPH y la TRE } \\
\text { no modifican } \\
\text { ni detienen } \\
\text { la progresión. }\end{array}$ \\
\hline $\begin{array}{l}\text { Afección } \\
\text { miocárdica }\end{array}$ & $\begin{array}{l}\text { Muy frecuente } \\
\text { en formas graves. }\end{array}$ & $\begin{array}{l}\text { Hipertrofia (el } 83 \%) . \\
\text { Dilatación. } \\
\text { Disfunción diastólica } \\
\text { en el } 50 \% .\end{array}$ & $\begin{array}{c}\text { Ecocardiograma. } \\
\text { Electrocardiograma: no demuestra } \\
\text { la hipertrofia debido a la } \\
\text { conductividad baja de } \\
\text { los glicosaminoglicanos. }\end{array}$ & $\begin{array}{c}\text { El TCPH y la TRE } \\
\text { a mejoran la hipertrofia } \\
\text { y la función ventricular. }\end{array}$ \\
\hline $\begin{array}{l}\text { Afección } \\
\text { coronaria }\end{array}$ & $\begin{array}{c}\text { Menos frecuente } \\
\text { que en otras } \\
\text { mucopolisacaridosis. }\end{array}$ & $\begin{array}{l}\text { Compromiso difuso } \\
\text { con isquemia } \\
\text { que lleva a } \\
\text { miocardiopatía } \\
\text { dilatada. }\end{array}$ & $\begin{array}{l}\text { TC multislice y perfusión } \\
\text { miocárdica para } \\
\text { el diagnóstico. } \\
\text { Electrocardiograma: } \\
\text { no evidencia isquemia. }\end{array}$ & $\begin{array}{l}\text { El efecto de la TCPH y TRE } \\
\text { sobre la lesión coronaria } \\
\text { dependerá de la gravedad } \\
\text { previa de esta afección. } \\
\text { Otras publicaciones dicen } \\
\text { que esto no influye. }\end{array}$ \\
\hline $\begin{array}{l}\text { Afección } \\
\text { arterial }\end{array}$ & $\begin{array}{l}\text { Dilatación de la raíz } \\
\text { aórtica en el } 38 \% \\
\text { de las formas graves. } \\
\text { HTA en el } 32 \% \text { de } \\
\text { los pacientes. }\end{array}$ & $\begin{array}{c}\text { Formas atenuadas } \\
\text { de estenosis. } \\
\text { Dolor y claudicación } \\
\text { en los miembros inferiores. } \\
\text { HTA: compromiso de } \\
\text { las arterias renales. }\end{array}$ & $\begin{array}{l}\text { Control de TA. } \\
\text { Sospecha clínica. } \\
\text { Ecocardiograma y } \\
\text { ecodoppler arterial. }\end{array}$ & No hay datos. \\
\hline $\begin{array}{l}\text { Hipertensión } \\
\text { pulmonar }\end{array}$ & Poco frecuente. & $\begin{array}{l}\text { Disnea. } \\
\text { Elevada morbimortalidad. }\end{array}$ & $\begin{array}{c}\text { Ecocardiograma. } \\
.\end{array}$ & No hay datos. \\
\hline $\begin{array}{l}\text { Disfunción } \\
\text { endotelial }\end{array}$ & $\begin{array}{l}\text { Riesgo aumentado } \\
\text { en MPS I. }\end{array}$ & $\begin{array}{c}\text { En adolescentes y } \\
\text { preadolescentes, la edad } \\
\text { vascular equivale a la de } \\
\text { un adulto de } 40 \text { años. }\end{array}$ & $\begin{array}{c}\text { Doppler de } \\
\text { vasos del cuello. }\end{array}$ & $\begin{array}{l}\text { Disminuye con TRE y TCPH, } \\
\text { aunque no se recupera la } \\
\text { función endotelial. }\end{array}$ \\
\hline
\end{tabular}

TCPH: trasplante de células precursoras hematopoyéticas; TRE: terapia de reemplazo enzimático; HTA: hipertensión arterial; TA: tensión arterial; MPS I: mucopolisacaridosis tipo I. 
- A futuro, habrá que evaluar el riesgo vascular, calculando la edad vascular mediante ecocardiograma doppler de los vasos del cuello y la velocidad de onda de pulso. ${ }^{11}$

\section{Evaluación de la vía aérea}

La obstruccion de la gran vía aérea es un grave problema. ${ }^{14}$ La hipertrofia de amígdalas palatinas y adenoides genera ronquido, obstrucción y apneas obstructivas. Se deben estudiar con polisomnografia de sueño y radiografía de cuello de perfil en posicion de olfateo. ${ }^{15,16}$

La amígdala-adenoidectomía mejora la ventilación y el efecto deletéreo de la hipoxia nocturna sobre el sistema nervioso central (SNC) y otros órganos. Debe ser cuidadosa, por posibles complicaciones cardíacas, sumadas a la presencia de infiltrados subglóticos. ${ }^{17}$

A más edad, se suman escoliosis y rigidez torácica, con incapacidad ventilatoria restrictiva. La acumulación de glicosaminoglicanos en la pared de la tráquea y los bronquios agrega incapacidad ventilatoria obstructiva, por lo que puede requerirse traqueotomía. ${ }^{18}$ Se ha descrito la infiltración instersticial pulmonar en el neonato. ${ }^{19}$ La vía aérea difícil debe evaluarse antes de una cirugía, ya que una traqueotomía puede evitar complicaciones en el posoperatorio. ${ }^{20,21}$

\section{Evaluación auditiva}

La patología recidivante del oído medio es frecuente y temprana. La reiteración de pesquisa auditiva anómala podría hacer sospechar una mucopolisacaridosis. Si hay pérdida auditiva progresiva, se debe reevaluar a $\operatorname{los} 6$, a $\operatorname{los} 9$ y a los 12 meses.

La hipoacusia es de tipo conductiva o mixta y algunas evolucionan a neurosensoriales. Los depósitos de glicosaminoglicanos ocurren primero en la membrana timpánica, luego en el oído interno y, finalmente, a nivel central.

Cuando hay una alteración de la conducción entre moderada y grave, se debe habilitar el canal auditivo para conservar el estímulo y la comunicación. El grado grave instalado ya no tiene indicación de tratamiento.

La indicación de dispositivos audioprotésicos, implantes cocleares y osteointegrados depende del nivel auditivo y del compromiso general del paciente. La metodología de evaluación audiológica debe incluir pruebas subjetivas o conductuales para la confirmación diagnóstica. ${ }^{22}$

\section{Salud espinal}

Se observa una deficiencia anterior y media del cuerpo vertebral, la clásica platispondilia con un pico o notch anterior, por osificación endocondral incompleta del cuerpo vertebral, ${ }^{23,24}$ vértebras trapezoidales o acuñadas, ${ }^{23}$ discos intervertebrales grandes en relación con vértebras hipoplásicas, presencia de abombamiento (bulging) posterior intracanal ${ }^{26,27}$ y estenosis de canal raquídeo a distintos niveles. La patología cervical es más rara en el tipo I que en el tipo IV y VI. La hipoplasia de apófisis odontoides, ${ }^{25}$ en algunos casos, asociada a inestabilidad cervical atlantoaxial, se detecta en el par radiológico cervical, en máxima flexión y extensión.

Las cifoescoliosis progresivas conducen a descompensación del tronco, inestabilidades y potencial compromiso neurológico. ${ }^{28,29}$ Los pacientes van desde estar asintomáticos a presentar compromiso neurológico secundario a una compresión medular. ${ }^{30,31}$

De 42 pacientes (atendidos en el Hospital Garrahan) con mucopolisacaridosis de distintos tipos (IV, VI y I) operados, el 55 \% se presentó con compromiso neurológico. Tenían hipoplasia odontoidea el $25 \%$, inestabilidad atlantoaxoidea el $21 \%$ y canal cervical estrecho por depósitos de glicosaminoglicanos el $21 \%$.

Con sospecha de estenosis cervical, al momento del examen clínico, se deben realizar estudios radiológicos, resonancia magnética estática y tomografías computadas preoperatorias. Se requiere la evaluación especializada. ${ }^{32}$ Se realizan, también, potenciales somatosensitivos, motores, algunas veces, urodinamias y polisomnografías.

La cirugía se realiza liberando compresiones y preservando las estructuras neurológicas, utilizando técnicas de descompresión posterior, en su gran mayoría, asociadas a artrodesis. ${ }^{32}$ Las fusiones profilácticas versus terapéuticas son controvertidas. La cirugía debe ser precoz cuando está indicada. ${ }^{33}$

La deformidad más frecuente es la cifosis con apex en la región L1 o L2, seguida por escoliosis, o combinadas. En los menores de 7 años con cifoescoliosis y curvas con ángulo inferior a 50 grados, se utiliza corsé toracolumbosacro termoplástico a medida, 22 h/día, que permite hacer actividad física o deportiva si no hay riesgo medular a nivel cervical o toracolumbar. Para las edades mayores, con curvas entre 50 y 70 grados o superiores, se indican fusiones posteriores instrumentadas. Con deformidad asociada 
a estenosis de canal y déficit neurológico, se realiza doble abordaje (anterior descompresión y artrodesis + posterior fusión instrumentada). El equipo quirúrgico incluye anestesiólogo y neurofisiólogo experimentados.

\section{Manifestaciones neurológicas}

La MPS I tiene compromiso evolutivo del SNC y periférico (SNP), variable según la forma clínica. Hay deterioro cognitivo, problemas de comportamiento, trastornos del sueño, hidrocefalia y compresión de la médula espinal, atrapamiento de raíces, nervios periféricos y pares craneales.

\section{Deterioro cognitivo}

En la FG, luego de un período de desarrollo normal, ocurre un estancamiento entre el primer y el segundo año de vida, seguido por un deterioro cognitivo rápido. ${ }^{34-36}$ Se pierden, aproximadamente, 20 puntos del coeficiente intelectual (CI) por año. ${ }^{37}$ La neurodegeneración involucra depósito de heparán sulfato, que actúa como matriz de neuroinflamación y sobreexpresión de gangliósidos con efecto neurotóxico, que induce dendritogénesis ectópica y sinaptogénesis anormal. ${ }^{38}$

Las FA no están totalmente exentas de problemas del desarrollo cognitivo. Por cada año que se retrase el tratamiento, habrá 9 puntos menos de CI. ${ }^{39}$ Algunas mutaciones (L238Q) favorecen manifestaciones psiquiátricas tardías, como aislamiento y depresión. ${ }^{40}$

No hay biomarcadores orientadores de neurodegeneración. Esta se mide mediante la evaluación cognitiva formal en el tiempo. Hasta los 3 años, se recomienda usar la escala Bayley-III, que brinda información de edad equivalente. En los mayores de 3 años, se puede medir el CI con las escalas de Wechsler. Para la conducta adaptativa, se recomienda la escala Vineland para todas las edades. ${ }^{34}$

El compromiso auditivo precoz puede, per se, afectar el lenguaje. Las nuevas neuroimágenes funcionales y su correlación con las funciones mentales superiores aún están en etapa de investigación. ${ }^{41}$ Para un mejor pronóstico cognitivo a largo plazo en FG, hay que trasplantar tempranamente. ${ }^{42}$

\section{Hidrocefalia}

La hidrocefalia es una complicación de relativa frecuencia en las mucopolisacaridosis, en especial, en MPS I y II, y, junto con los depósitos de glicosaminoglicanos en los diferentes planos, contribuye a la macrocefalia. Se presenta con lento deterioro neurológico, decaimiento progresivo y, raramente, edema de papila, cefalea y vómitos.

El 80-85\% son comunicantes. ${ }^{43}$ La dinámica de la circulación del líquido cefalorraquídeo (LCR) se altera por depósito de glicosaminoglicanos con obstrucción de los espacios subaracnoideos y granulaciones de Pacchioni, con reducción del drenaje venoso.

En los niños, se corrige cuando se requiere, con derivación ventrículo-peritoneal o ventrículoatrial o lumboperitoneal para acortar el recorrido subcutáneo. Los glicosaminoglicanos pueden obstruir la derivación.

El depósito de glicosaminoglicanos en la sustancia blanca y ganglios de la base produce destrucción tisular, compresión de los vasos del parénquima, dilatación de los espacios perivasculares y subaracnoideos, que conduce a la atrofia cerebral con dilatación pasiva de los ventrículos y mimetiza la hidrocefalia. Esta no requiere tratamiento.

\section{Compresión medular}

El depósito de glicosaminoglicanos en la duramadre y los ligamentos causa compresión medular y mielopatía. Su detección y corrección quirúrgica precoz evitan el daño medular irreversible. Los signos iniciales, leves e insidiosos (debilidad progresiva, torpeza, fatiga, hiperreflexia, dolor y parestesias) pueden confundirse con signos de la enfermedad osteoarticular. ${ }^{37,38}$

\section{Diagnóstico}

Se realiza demostrando en el paciente, con signos y síntomas sugestivos, la deficiencia de la enzima alfa-L-iduronidasa en leucocitos purificados o en gotas de sangre en papel de filtro. También puede realizarse la pesquisa neonatal. ${ }^{1,8,9}$

Los métodos disponibles actualmente hacen prescindible medir la actividad enzimática en cultivo de fibroblastos. En la Tabla 2, se muestran ventajas y limitaciones de los métodos bioquímicos disponibles. El estudio molecular no es imprescindible para el diagnóstico, pero es necesario para indicar el tratamiento.

\section{Tratamientos disponibles}

Los tratamientos específicos son el trasplante alogénico de células progenitoras hematopoyéticas (TCPH) o la terapia de 
reemplazo enzimático (TRE). El consenso europeo remite a un equipo de expertos la decisión terapéutica, que es una excelente recomendación para nuestro medio. ${ }^{44}$

El TCPH es el tratamiento de elección para la FG para reemplazar el sistema monocíticomacrofágico deficiente por el del donante, que secreta la enzima faltante. Ofrecido a $<2$ años y con un $\mathrm{CI}>70$, puede detener el deterioro neurocognitivo progresivo y el daño sobre otros órganos. ${ }^{8,44}$ Los mayores de 2 años y con $\mathrm{CI}<70$ tienen pobre beneficio con el TCPH.
La edad es la variable más importante. Trasplantando antes de los 12 meses, se logra el mejor pronóstico cognitivo y, antes de los 9 meses, el desarrollo neurocognitivo alcanzado puede ser normal. ${ }^{42,45-48}$

Desde el primer TCPH en 1981 hasta la fecha, se han trasplantado más de 500 pacientes. En la Argentina, entre 2009 y 2018, diecisiete pacientes recibieron $20 \mathrm{TCPH}^{*}\left({ }^{*}\right.$ datos aportados por el Instituto Nacional Central Único Coordinador de Ablación e Implante-INCUCAI-). Un 25\% tendrá un donante familiar; el resto requerirá una

Tabla 2. Métodos de diagnóstico

\begin{tabular}{lll}
\hline Tipo de muestra & Ventajas & Limitaciones \\
\hline & Glicosaminoglicanos en orina & \\
\hline
\end{tabular}

\section{Cuantitativo}

Medir la concentración

de glicosaminoglicanos

en orina corregido

por la creatinina.
- Orina de $24 \mathrm{~h} \quad$ • Es útil como método de pesquisa para aquellas mucopolisacaridosis que presentan altas concentraciones de glicosaminoglicanos en orina. - El ensayo puede ser usado para monitorear la evolución de la enfermedad o la respuesta al tratamiento.
- No es específico para heparán y dermatán sulfato.

- Algunos pacientes pueden excretar cantidades normales de glicosaminoglicanos o levemente aumentados.

- Los niveles de corte deben estar establecidos acorde a la edad del paciente. La concentración de glicosaminoglicanos

disminuye conforme aumenta la edad del paciente.

- Se pueden obtener resultados falsos positivos si la orina presenta una concentración de creatinina elevada o negativos si la orina es muy diluida.

- La muestra es difícil de obtener en pacientes que no controlan esfínteres (niños pequeños o con retraso mental).

- El envío de muestras hasta un centro de referencia.

- Un resultado negativo puede hacer que no se siga estudiando a un paciente.

\begin{tabular}{|c|c|c|c|}
\hline $\begin{array}{l}\text { Cualitativo } \\
\text { Identifica } \\
\text { específicamente el tipo } \\
\text { de glicosaminoglicano } \\
\text { presente en la orina, } \\
\text { lo que permite tipificar } \\
\text { el tipo de } \\
\text { mucopolisacaridosis } \\
\text { que tiene el paciente. }\end{array}$ & - Orina de 24 h. & $\begin{array}{l}\text { - Se puede observar un patrón } \\
\text { específico de excreción para distintas } \\
\text { mucopolisacaridosis, aun en casos } \\
\text { en los que la cuantificación de } \\
\text { glicosaminoglicanos fue normal. }\end{array}$ & $\begin{array}{l}\text { - El perfil obtenido no permite discriminar entre MPS I y II. } \\
\text { - La muestra es difícil de obtener en pacientes que } \\
\text { no controlan esfínteres (niños pequeños o con retraso mental). } \\
\text { - Se requiere un laboratorio especializado para su realización. }\end{array}$ \\
\hline \multicolumn{4}{|c|}{ Ensayo de actividad enzimática de alfa-iduronidasa } \\
\hline Leucocitos & $\begin{array}{l}\text { - } 10 \mathrm{ml} \text { de } \\
\text { sangre } \\
\text { heparinizada. }\end{array}$ & $\begin{array}{l}\text { - Método altamente sensible y específico. } \\
\text { - Una vez acondicionada la muestra, } \\
\text { se puede conservar mucho tiempo. }\end{array}$ & $\begin{array}{l}\text { - La muestra debe llegar al laboratorio dentro de las } 24 \mathrm{~h} \\
\text { de haber sido obtenida. } \\
\text { - El volumen de muestra requerido es elevado, sobre todo, } \\
\text { en pacientes pediátricos. } \\
\text { - Se requiere un laboratorio especializado para su realización. }\end{array}$ \\
\hline Fibroblastos & - Biopsia de piel. & $\begin{array}{l}\text { - Se pueden realizar múltiples ensayos } \\
\text { de una misma muestra. } \\
\text { - Una vez acondicionada la muestra, } \\
\text { se puede conservar mucho tiempo. }\end{array}$ & $\begin{array}{l}\text { - Toma de muestra invasiva. } \\
\text { - El desarrollo del cultivo de fibroblastos demanda semanas hasta } \\
\text { estar en condiciones para hacer el ensayo enzimático, } \\
\text { lo que demora el resultado. } \\
\text { - El transporte puede alterar la muestra. } \\
\text { - Se requiere un laboratorio especializado para su realización. }\end{array}$ \\
\hline GSPF & $\begin{array}{l}\text { - Sangre entera } \\
\text { impregnada en } \\
\text { papel (el mismo } \\
\text { que se utiliza } \\
\text { para pesquisa } \\
\text { neonatal). }\end{array}$ & $\begin{array}{l}\text { - Toma de muestra mínimamente } \\
\text { invasiva. } \\
\text { - Fácil transporte. } \\
\text { - Permite realizar varios ensayos } \\
\text { sobre una misma muestra. } \\
\text { - Permite hacer estudios a gran escala. } \\
\text { - Buena correlación de resultados } \\
\text { obtenidos en leucocitos. }\end{array}$ & $\begin{array}{l}\text { - Se requiere un laboratorio especializado para su realización. } \\
\text { - Los resultados obtenidos deben ser confirmados en leucocitos o } \\
\text { con el estudio genético. }\end{array}$ \\
\hline
\end{tabular}

GSPF: gota de sangre en papel de filtro. 
búsqueda en registros internacionales.

Puede haber hasta un $30 \%$ de pérdida del injerto, pero los acondicionamientos mieloablativos, basados en busulfán han mejorado este aspecto. Con el esquema busulfán/ fludarabina, se reportan sobrevidas $>90 \% .42,44,45,49$

La sobrevida global (SG) a los 5 años es del 74-95 \%, y la libre de eventos, del 63-90 \%, dependiendo del tipo de donante y la fuente de TCPH. ${ }^{46,47}$ La mortalidad relacionada con el trasplante, del $10 \%$ al $25 \%$, es, principalmente, por causas infecciosas virales, enfermedad injerto contra huésped (EICH), complicaciones pulmonares, hemorragias y falla múltiple de órgano. ${ }^{46,49}$

La sangre de cordón umbilical (SCU) ha mostrado mayor probabilidad de lograr quimerismo del donante $(\mathrm{p}=0,39)$ y niveles enzimáticos normales $(\mathrm{p}=0,07)(\mathrm{el} 92 \%$ y el $98 \%$, respectivamente) comparada con otras fuentes. La SG con SCU (identidad 6/6), el $81 \%$, es comparable con el trasplante de donante familiar idéntico. Sería una fuente preferencial por la rápida disponibilidad y tener mayor población de $\mathrm{CPH}$ primitivas. El período de aplasia es más prolongado, con el consiguiente mayor riesgo de reactivaciones virales, y se tiene una sola donación por donante. Los resultados con SCU (SG $90 \%$ ) son mejores que con médula ósea o sangre periférica. Podrían estar influidos por los cuidados de soporte, acondicionamientos y alto grado de compatibilidad por ser de la última década. ${ }^{45-47,49}$

Las opciones para un TCPH serían las siguientes:

1. Donante familiar idéntico (hermano, con estudios moleculares).

2. Donante no relacionado (DNR) $10 / 10$ o SCU 6/6 (antígenos leucocitarios humanos -human leucocyte antigen; HLA, por sus siglas en inglésen alta resolución).

La actividad enzimática alcanza valores comparables con los del donante en los primeros 3 meses. Se logra, aproximadamente, el $50 \%$ del valor normal con donante portador y el $100 \%$ con donante no afectado. ${ }^{49}$

Puede persistir el compromiso en algunos órganos, dependiendo de la carga de enfermedad previa. Mejoran la hepatoesplenomegalia, los síntomas ventilatorios y la hipertrofia miocárdica, aunque las alteraciones valvulares persisten. Mejora la opacidad corneal, pero puede ocurrir degeneración retiniana. La audición también mejora, aun con un componente neurosensorial.
La mayoría queda con estatura corta. Las displasias óseas persisten. Mejora la displasia odontoidea y la estabilidad cervical, la movilidad articular de las extremidades superiores, más que las inferiores. Puede haber retraso puberal y fertilidad reducida. ${ }^{42,43,46} \mathrm{El}$ deterioro cognitivo mejora 6-12 meses postrasplante porque requiere la presencia de los macrófagos del donante transportadores de la enzima en la microglía y la tasa de reemplazo es lenta. ${ }^{42,45,49,50}$

El pronóstico del TCPH a largo plazo depende de la edad, el estado clínico basal y el quimerismo completo. Es importantísimo el diagnóstico temprano, la derivación oportuna y la aceptación rápida en centros de trasplante con experiencia. ${ }^{42,45,49,50} \mathrm{El}$ seguimiento debe hacerlo un equipo multidisciplinario e interdisciplinario, con participación activa, al menos, de 1 año del equipo que trasplante.

La TRE con laronidasa humana recombinante es para las FA, no para las FG, porque no atraviesa la barrera hematoencefálica (BHE) ${ }^{45}$ El mejor resultado terapéutico depende de la edad, la gravedad y el grado y tipo de compromiso clínico de cada paciente. ${ }^{45}$

Con TCPH, se obtiene mejor control metabólico que con TRE. ${ }^{51}$ No corrige las afecciones en el SNC, hueso, válvulas cardíacas ni córneas. La TRE pretrasplante mejora la función respiratoria y la miocardiopatía. No aumenta el riesgo de reacciones inmunomediadas, no afecta negativamente al injerto ni promueve la EICH. Se reportó una mejoría de las funciones cognitivas con TRE postrasplante, probablemente, por la mejoría de las demás manifestaciones clínicas. ${ }^{52}$

La TRE modifica la historia natural de la enfermedad en las FA, mejora la hepatoesplenomegalia, el grosor de la piel, la función respiratoria y la miocardiopatía, pero no revierte el rango anormal de la movilidad articular ya establecida y afecciones como la disostosis, la valvulopatía y la opacidad corneal. La TRE temprana, en FA, antes de que se instale la cascada inflamatoria posterior al depósito de glicosaminoglicanos, evita algunas de las manifestaciones. La indicación difiere, entonces, de los criterios establecidos en 2008 para tratar las FA. ${ }^{1}$ Debe comenzar cuanto antes.

Se comunicó una mejor evolución con 12 años de seguimiento de un paciente que inició TRE a los 5 meses, comparado con su hermana que la comenzó a los 5 años. El hermano menor mantuvo facies normal, menos lesiones óseas, de las articulaciones y del miocardio, creció en 
percentilo 97, y la hermana en el 10, y alcanzó un CI 100, y la hermana $70 .{ }^{53}$ El beneficio de TRE parece mínimo en FG no trasplantados. ${ }^{54}$ Se sugiere que un grupo de expertos evalúe individualmente su indicación. ${ }^{55}$

\section{CONCLUSIONES}

El diagnóstico y tratamiento tempranos modifican la evolución. Idealmente, el seguimiento estará a cargo de un equipo interdisciplinario.

\section{REFERENCIAS}

1. Grupodetrabajodeenfermedadespocofrecuentes.Consenso de diagnóstico y tratamiento de la mucopolisacaridosis tipo I. Arch Argent Pediatr. 2008; 106(4):361-8.

2. ClarkeLA, Giugliani R, Guffon N, JonesSA, etal.Genotype $\square$ phenotype relationships in mucopolysaccharidosis type I (MPS I): Insights from the International MPS I Registry. Clin Genet. 2019; 96(4):281-9.

3. Izumi Y, Saito T, Sato S, Shimizu W. Myocardial Alterations in a Patient with Mucopolysaccharidosis Type I. Eur Heart J. 2018; 39(21):1863.

4. Soliman OI, Timmermans RG, Nemes A, Vletter WB, et al. Cardiac abnormalities in adults with the attenuated form of mucopolysaccharidosis type I. J Inherit Metab Dis. 2007; 30(5):750-7.

5. Watanabe N, Anagnostopoulos PV, Azakie A. Aortic stenosis in a patient with Hurler's syndrome after bone marrow transplantation. Cardiol Young. 2011;21(3):349-50.

6. Robinson CR, Roberts WC. Outcome of Combined Mitral and Aortic Valve Replacement in Adults with Mucopolysaccharidosis (the Hurler Syndrome). Am J Cardiol. 2017; 120(11):2113-8.

7. Dangel JH. Cardiovascular Changes in Children with Mucopolysaccharide Storage Diseases and Related Disorders-Clinical and Echocardiographic Findings in 64 Patients. Eur J Pediatr. 1998; 157(7):534-8.

8. Muenzer J, Wraith E, Clarke LA. Mucopolisaccharidosis I: management and treatment guidelines. Pediatrics. 2009; 123(1):19-29.

9. Martins AM. Introduction to Brazilian Guidelines tod, treatment, and monitoring for Gaucher disease, Fabry disease, Mucopolysaccharidosis I, and Pompe disease. J Pediatr. 2009; 155(4 Suppl):S9.

10. Muñoz-Rojas MV, Bay L, Sanchez L, Van KuijckM, et al. Clinical manifestations and treatment of mucopolysaccharidosis type i patients in Latina America as compared with the rest of the world. J Inherit Metab Dis. 2011; 34(5):1029-37.

11. Kelly AS, Metzig AM, Steinberger J, Braunlin EA. Endothelial function in children and adolescents with mucopolysaccharidosis. J Inherit Metab Dis. 2013;36(2):2215.

12. Wang R Y, Rudser KD, Dengel DR, Braunlin EA, et al. The Carotid Intima-Media Thickness and Arterial Stiffness of Pediatric Mucopolysaccharidosis Patients are Increased Compared to Both Pediatric and Adult Controls. Int J Mol Sci. 2017; 18(3):637.

13. LealGN, DePaula AC, LeoneC, Kim CA.Echocardiographic study of paediatric patients with mucopolysaccharidosis. Cardiol Young. 2010; 20(3):254-61.

14. Moretto A, Bosatra MG, Marchesini L, Tesoro S. Anesthesiological risks in mucopolysaccharidoses. Ital J Pediatr. 2018; 44(Suppl 2):116.
15. Solaiman SS, Rifkin DS, Rao H. Sleep Apnea in Hurler Syndrome: Looking Beyond the Upper Airway. J Clin Sleep Med. 2016; 12(10):1423-4.

16. Wooten WI 3rd, Muenzer J, Vaughn BV, Muhlebach MS. Relationship of Sleep to Pulmonary Function in Mucopolysaccharidosis II. J Pediatr. 2013; 162(6):1210-5.

17. Semenza GL, Pyeritz RE. Respiratory complications of mucopolysaccharide storage disorders. Medicine(Baltimore). 1988; 67(4):209-19.

18. Marcus CL. Sleep-disordered breathing in children. Am J Respir Crit Care Med. 2001; 164(1):16-30.

19. Bush D, Sremba L, Lomax K, Lipsett J, et al. Neonatal Onset Interstitial Lung Disease as a Primary Presenting Manifestation of Mucopolysaccharidosis Type I. JIMD Rep. 2019; 43:71-7.

20. Allen JL. Treatment of respiratory system (not just lung!) abnormalities in mucopolysaccharidosis I. J Pediatr. 2004; 144(5):561-2.

21. Shinhar SY, Zablocki H, Madgy DN. Airway management in mucopolysaccharide storage disorders. Arch Otolaryngol Head Neck Surg. 2004; 130(2):233-7.

22. Gökdoğan Ç, Altinyay Ş, Gökdoğan O, Tutar H, et al. Audiologic evaluations of children with mucopolysaccharidosis. Braz J Otorhinolaryngol. 2016; 82(3):281-4

23. White KK. Orthopaedic aspects of mucopolysaccharidoses. Rheumatology (Oxford). 2011; 50(Suppl 5):v26-33.

24. Field RE, Buchanan JAF, Copplemans MGJ, Aichroth PM. Bone marrow transplantation in Hurlers syndrome: effect on skeletal development. J Bone Joint Surg Br. 1994;76(6):97581.

25. Langer L Jr, Carey L. The roentgenographic features of the KS mucopolysaccharidosis of Morquio (MorquioBrailsford's disease). Am J Roentgenol Radium Ther Nucl Med. 1966; 97(1):1-20.

26. Swischuk LE. The beaked, notched, or hooked vertebra: Its significance in infants and young children. Radiology. 1970; 95(3):661-4.

27. Tandon V, Williamson JB, Cowie RA, Wraith JE. Spinal problems in mucopolysaccharidosis I (Hurler syndrome). J Bone Joint Surg Br. 1996; 78(6):938-44.

28. Holte DC, Rogers MA, Ransford AO, Crockard HA, et al. Thoracic spinal cord compression in Morquio-Brailsford's disease: Report of a case and review of the literature. NeuroOrthopaedics. 1994; 16:31-7.

29. Kulkarni MV, Williams JC, Yeakley JW, Andrews JL, et al. Magnetic resonance imaging in the diagnosis of the craniocervical manifestations of the mucopolysaccharidoses. Magn Reson Imaging. 1987; 5(5):317-23.

30. Hughes DG, Chadderton RD, Cowie RA, Wraith JE, et al. MRI of the brain and craniocervical junction in Morquio's disease. Neuroradiology. 1997; 39(5):381-5.

31. Ransford A, Crockard H, Stevens J, Modaghegh S. Occipitoatlanto-axial fusion in Morquio-Brailsford Syndrome. J Bone Joint Surg Br. 1996; 78(2):307-13.

32. KachurE, Del Maestro R. Mucopolysaccharidosis and spinal cord compression: case report and review of the literature with implications of bone marrow transplantation. Neurosurgery. 2000; 47(1):223-9.

33. Sostrin RD, Hasso AN, Peterson DI, Thompson JR. Myelographic features of mucopolysaccharidosis: a new sign. Radiology. 1977; 125(2):421-4.

34. Shapiro EG, Escolar ML, Delaney KA, Mitchell JJ. Assessments of neurocognitive and behavioral function in the mucopolysaccharidoses. Mol Genet Metab. 2017; 122(Suppl:8)-16.

35. Shapiro EG, Nestrasil I, Rudser K, Delaney $K$, et al. Neurocognition across the spectrum of 
mucopolysaccharidosis type I: age, severity, and treatment. Mol Genet Metab. 2015; 116(1-2):61-8.

36. KrivitW,PetersC,ShapiroEG. Bonemarrow transplantation as effective treatment of central nervous system disease in globoid cell leukodystrophy, metachromatic leukodystrophy, adrenoleukodystrophy, mannosidosis, fucosidosis, aspartylglucosaminuria, Hurler, MaroteauxLamy and Sly syndromes, and Gaucher disease type III. Curr Opin Neurol. 1999;v12(2):167-76.

37. Krenzlin H, Ta-Chih T, Lampe C, Knuf M, et al. Standalone cráneo-cervical decompresiion is feasible in children with MPS type I, IVA and VI. Spine J. 2018; 18(8):1455-9.

38. Vitner EB, Platt FM, Futerman AH. Common and uncommon pathogenic cascades in lysosomal storage diseases. J Biol Chem. 2010; 285(27):20423-7.

39. Van der Lee JH, Morton J, Adams HR, Clarke L, et al. Cognitive endpoints for therapy development for neuronopathic mucopolysaccharidoses: results of a consensus procedure. Mol Genet Metab. 2017; 121(2):70-9.

40. Ahmed A, Whitley CB, Cooksley R, Rudser K, et al. Neurocognitive and neuropsychiatric phenotypes associated with the mutation L238Q of the $\alpha$-L-iduronidase gene in Hurler-Scheie syndrome. Mol Genet Metab. 2014; 111(2):123-7.

41. Shapiro EG. Longitudinal studies of brain structure and function in MPS disorders: a study of the lysosomal disease network. Mol Genet Metab. 2010; 99(2)s34.

42. Parini R, Deodato F, Di Rocco M, Lanino E, et al. Open Issues in Mucopolysaccharidosis type I-Hurler. Orphanet J Rare Dis. 2017; 12(1):112.

43. Aldenhoven M, Wynn R, Orchard P, O'Meara A, et al. Long-term outcome of Hurler Syndrome patients after hematopoietic cell transplantation: an international multicenter study. Blood. 2015; 125(13):2164-72.

44. Martins AM, Dualibi AP, Norato D, Takata ET, et al. Guidelines for the Management of Mucopolysaccharidosis Type I. J Pediatr. 2009; 155(4 Suppl):S32-46.

45. De Ru MH, Boelens JJ, Das AM, Jones SA, et al. Enzyme replacement therapy and/or hematopoietic stem cell transplantation at diagnosis in patients with mucopolysaccharidosis type I: results of a European consensus procedure. Orphanet J Rare Dis. 2011; 6:55.

46. Boelens JJ, Prasad VK, Tolar J, Wynn RF, et al. Current international perspectives on hematopoietic stem cell transplantation for inherited metabolic disorders. Pediatr Clin North Am. 2010; 57(1):123-45.

47. Aldenhoven M, Jones S, Bonney D, Borrill RE, et al. Hematopoietic cell transplantation for Mucopolysaccharidosis patients is safe and effective: Results after implementation of international Guidelines. Biol Marrow Transplant. 2015; 21(6)1106-9.

${ }^{4}$. Boelens JJ, Aldenhoven M, Purtill D, Ruggeri A, et al. Outcomes of transplantation using various hematopoietic cell sources in children with Hurler syndrome after myeloablative conditioning. Blood. 2013; 121(19):3981-7.

49. Poe MD, Chagnon SL, Escolar ML. Early treatment is associated with improved cognition in Hurler syndrome. Ann Neurol. 2014; 76(5):747-53.

50. Aldenhoven M, Boelens JJ, De Koning T. The clinical outcome of Hurler syndrome after stem cell transplantation. Biol Blood Marrow Transplant. 2008; 14(5):485-98.

51. Wynn RF, Wraith JE, Mercer J, O'Meara A, et al. Improved metabolic correction in patients with lysosomal storage disease treated with hematopoietic stem cell transplant compared with enzyme replacement therapy. J Pediatr. 2009; 154(4):609-11.

52. Eisengart JB, Rudser KD, Tolar J, Orchard PJ, et al. Enzyme replacement is associated with better cognitive outcomes after transplant in Hurler syndrome. J Pediatr. 2013; 162(2):375-80.e1.

53. Gabrielli O, Clarke LA, Ficcadenti A, Santoro L, et al. 12 year follow up of enzyme-replacement therapy in two siblings with attenuated mucopolysaccharidosis I: the important role of early treatment. BMC Med Genet. 2016; 17:19.

${ }^{5}$ 4. Yano S, Moseley K, Pavlova Z. Postmortem studies on a patient with mucopolysaccharidosis type I: histopathological findings after one year of enzyme replacement therapy. J Inherit Metab Dis. 2009; 32(Suppl 1):S53-7.

55. Pasad VK, Kurtzberg j. Transplant outcome in mucopolysaccharidoses. Semin Hematol. 2010; 47(1):59-69 